\title{
Molecular typing of Acinetobacter baumannii by automated repetitive-sequence-based PCR
}

\author{
Giuseppe Russello', Silvana Perin', Fabrizio Marini', Serena Barberis², Gabriele M. Perotti², \\ Claudio Farina' \\ I UOC Microbiologia e Virologia, AO "Ospedale San Carlo Borromeo", Milano \\ 2 Direzione Medica di Presidio, AO "Ospedale San Carlo Borromeo", Milano
}

Key words: Acinetobacter baumannii, Outbreak, Ribotyping

\section{Caratterizzazione molecolare di Acinetobacter baumannii con metodo di rep-PCR}

\section{SUMMARY}

Acinetobacter baumannii has been increasingly reported as a significant causative organism of various nosocomial infections. Here we describe three different outbreaks of multidrug resistant A. baumannii started in the Intensitive Care Unit and then involving other wards of San Carlo Borromeo hospital in Milan, Italy. In order to characterize the clinical strains isolates, molecular typing using semi-automated repetitive-sequence-based PCR (rep-PCR) was performed.Among the sixty-one strains analyzed, three main cluster $(\mathrm{Cl}, \mathrm{C2}, \mathrm{C} 3)$ were detected: $\mathrm{Cl}$ included six indistinguishable strains, $\mathrm{C} 2$ five and $\mathrm{C} 3$ thirty. No correlation was observed between chemosensitivity and ribotyping pattern and an high rate of carbapenems resistance was founded.

\section{INTRODUZIONE}

I batteri del genere Acinetobacter sono microrganismi Gram negativi a bassa virulenza. Tuttavia il loro ruolo quali responsabili di infezioni nosocomiali è assolutamente ingravescente a far corso dagli ultimi 40 anni $(1-2,13)$, a motivo della sua capacità di sopravvivere a lungo sulle superfici piane e di acquisire caratteri di multi-antibioticoresistenza (7).

Molti episodi epidemici nosocomiali sostenuti da Acinetobacter spp. sono ormai segnalati nella letteratura scientifica, in particolare in reparti di Terapia Intensiva o per Grandi Ustionati, conseguenti soprattutto alla contaminazione delle mani del personale di assistenza e di cura e dei presidi medici. La terapia delle malattie da infezione da Acinetobacter spp. è resa complessa dall'emergenza e, poi, dalla rapida diffusione di ceppi multidrug-resistant (MDR). In particolare, significativo è l'incremento degli stipiti resistenti ai carbapenemi per l'acquisizione di geni che codificano per carbapenem-hydrolyzing class $D \beta$-lactamase (CHDL). Tale resistenza acquisita comporta che, in questi casi, la strategia terapeutica consideri la possibilità di utilizzare Ampicillina-Sulbactam e Colistina (3)

È stato condotto uno studio osservazionale retrospettivo sui pazienti colonizzati ed affetti da malattia da infezione da $A$. baumannii ricoverati presso l'AO "Ospedale San Carlo Borromeo" tra novembre 2009 e agosto 2010, utilizzando la tecnica di tipizzazione molecolare rep-PCR e di caratterizzazione fenotipica del pattern di antibioticoresistenza.

\section{MATERIALI E METODI}

\section{Ceppi microbici}

Tutti i ceppi di Acinetobacter spp. studiati sono stati isolati consecutivamente da 61 pazienti ricoverati presso l'AO "Ospedale San Carlo Borromeo" di Milano in un periodo di otto mesi (novembre 2009 - agosto 2010).

L'identificazione microbica è stata effettuata sulla base delle caratteristiche morfologiche (colorazione di Gram) e biochimiche (API 20 NE e VITEK2 cards GN-ID, bioMérieux, La Balme les Grottes, France) in accordo con le istruzioni del produttore. I ceppi microbici sono stati conservati in sangue defibrinato di montone a $-80^{\circ} \mathrm{C}$. Successivamente gli stessi microrganismi sono stati valutati con il metodo di ribotipizzazione rep-PCR e di caratterizzazione del profilo di suscettibilità agli antibiotici. Il ceppo di riferimento ATCC 19606 è stato utilizzato come controllo per i test di suscettibilità.

Sono stati utilizzati i ceppi di controllo di $\mathrm{A}$. baumannii European clone I, II e III, gentilmente forniti da E. Carretto, IRCSS-Policlinico "S. Matteo", Pavia.

\section{Test di chemioantibioticosensibilità}

La suscettibilità antibiotica è stata valutata con il metodo di agar diffusione sec. Kirby-Bauer su terreno Mueller Hinton, in accordo con i criteri del Clinical and Laboratory Standards Institute

\section{Corresponding author: Claudio Farina}

UOC Microbiologia e Virologia, AO "Ospedale San Carlo Borromeo"

Via Pio II, 3 - 20153 Milano - Tel.: +39 240222456 - Fax: +39 240222829

E-mail: farina.claudio@sancarlo.mi.it 
(CLSI, M100-S17).

Gli antibiotici testati sono stati: ampicillina $(10 \mu \mathrm{g})$, amoxicillina/acido clavulanico $(20+10$ $\mu \mathrm{g})$, piperacillina $(100 \mu \mathrm{g})$, piperacillina/tazobactam $(100+10 \mu \mathrm{g})$, ceftazidime $(30 \mu \mathrm{g})$, ceftriaxone $(30 \mu \mathrm{g})$, gentamicina $(10 \mu \mathrm{g})$, ciprofloxacina $(5 \mu \mathrm{g})$, cotrimossazolo $(1.25+23.7 \mu \mathrm{g})$, amikacina $(30 \mu \mathrm{g})$, cefepime $(30 \mu \mathrm{g})$, cefazolina $(30 \mu \mathrm{g})$, imipenem $(10 \mu \mathrm{g})$, meropenem $(10 \mu \mathrm{g})$, colistina $(10 \mu \mathrm{g})$, ampicillina-sulbactam $(10+10 \mu \mathrm{g})$, aztreonam $(30 \mu \mathrm{g})$, e tobramicina $(10 \mu \mathrm{g})$.

La Minima Concentrazione Inibente per tigeciclina e colistina é stata determinata con il metodo Etest (bioMérieux s.a) in accordo con le indicazioni del produttore.

\section{Tipizzazione molecolare}

È stata utilizzata, in accordo con le indicazioni del produttore, la tecnica di rep-PCR (repetitive sequence-based polymerase chain reaction) automatizzata (DiversiLab ${ }^{\circledR}$, bioMérieux Inc., St. Louis, USA), che permette di classificare gli agenti microbici, sulla base dei loro pattern di fingerprinting, a livello di subspecie. È un metodo altamente riproducibile che consente di discriminare ceppi clonali differenti, per tracciare la fonte e la diffusione di focolai epidemici. Essa prevede quattro passaggi successivi per generare fingerprint unici per ciascun campione microbico: estrazione del DNA da colture isolate, amplificazione mediante rep-PCR, separazione dei frammenti amplificati ed analisi dei dati.

L'analisi dei dati é stata effettuata con il software DiversiLab ${ }^{\mathrm{TM}}$, version 3.4, che utilizza il coefficiente di correlazione di Pearson per definire dendrogrammi, elettroferogrammi, immagini virtuali e scatter plots. I ceppi sono considerati "indistinguibili" se la similarità è $>97 \%$ senza differenze a livello dei pattern di fingerprinting, "simili" se la similarità è $>95 \%$ ed una differenza a livello di pattern e "differenti" se la similarità è $<95 \%$ con due o più differenze.

\section{RISULTATI}

La Figura I illustra i risultati dell'analisi di repPCR e del gel virtuale. Sono stati individuati 19 pattern diversi. L'analisi del dendrogramma ha evidenziato l'esistenza di due cluster principali $\left(A_{1}\right.$ e $\left.A_{2}\right)$, caratterizzati da una similarità media di $62.7 \%$.

Il primo cluster include solo uno stipite di Acinetobacter spp. Il cluster $\mathrm{A}_{2}$ include 18 pattern differenti e, in particolare, può essere suddiviso in tre subcluster principali (C1, C2, and $\mathrm{C} 3$ ).

$\mathrm{C} 1$ include sei ceppi indistinguibili che presentano un'average similarity ratio di $96.1 \%$, senza differenze nelle bande. Questi ceppi sono stati

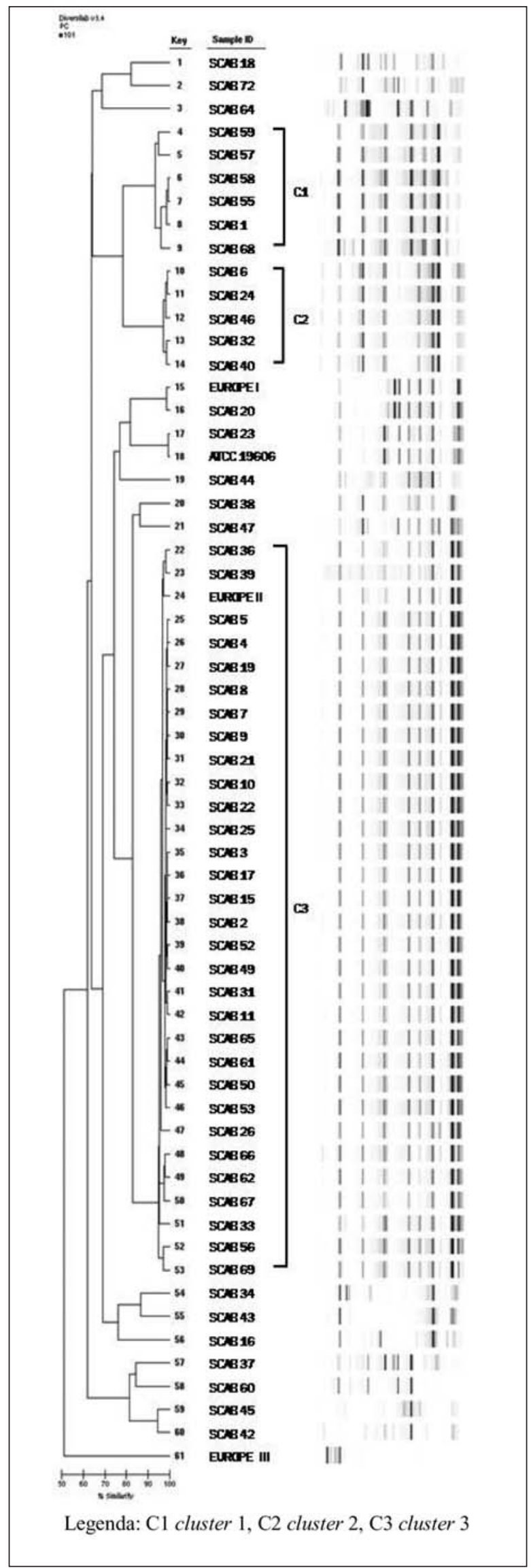

Figura I. Analisi rep-PCR. Da destra a sinistra è possibile osservare: elettroferogramma, campioni testati, cluster riscontrati e gel virtuale. 
Tabella I.Test di chemioantibioticosensibilità in vitro sui ceppi di Acinetobacter baumannii

Cluster AMC TZP CRO FEP CAZ AZT SAM G AK CIP SXT IP MEM TOB CS TCG \begin{tabular}{llllllllllllllll}
$R$ & $R$ & $R$ & $R$ & $R$ & $R$ & $S$ & $R$ & $R$ & $R$ & $S$ & $S$ & $S$ & $R$ & $S$ & 2 \\
\hline
\end{tabular}

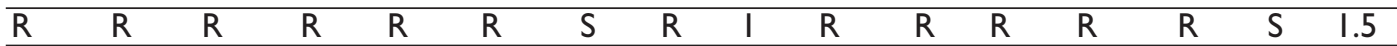

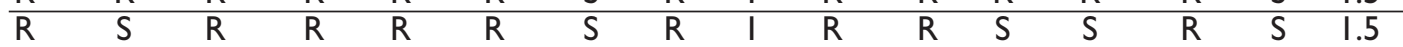

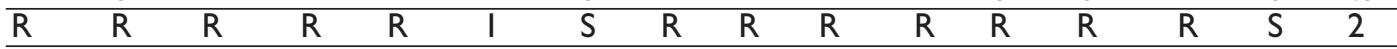

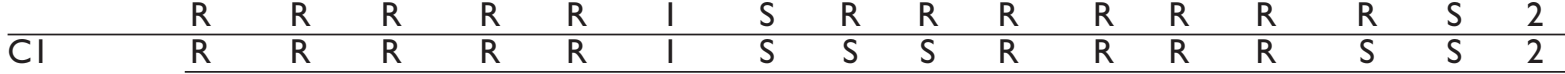

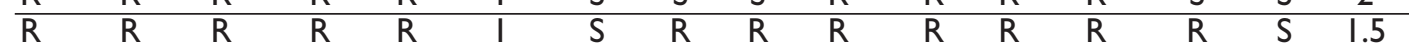

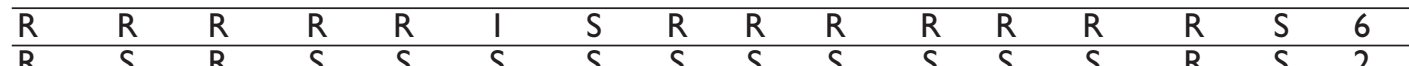

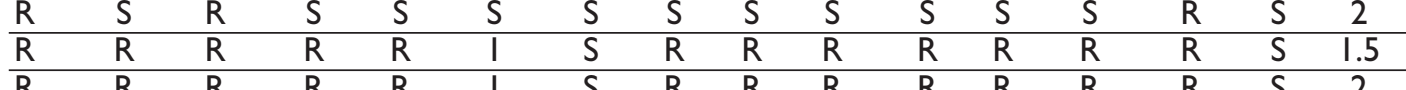

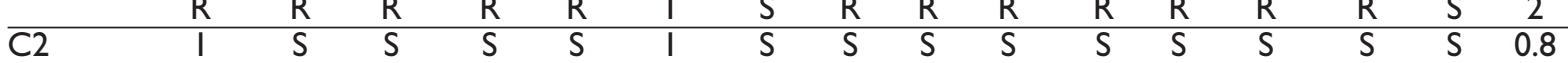

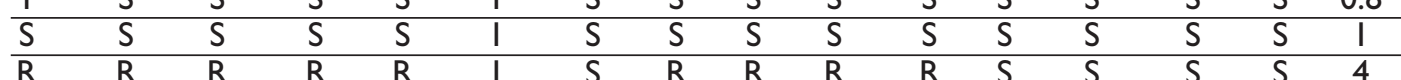

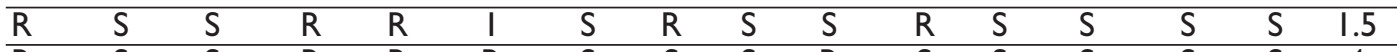
\begin{tabular}{llllllllllllllll}
\hline$R$ & $\mathrm{~S}$ & $\mathrm{~S}$ & $\mathrm{R}$ & $\mathrm{R}$ & $\mathrm{R}$ & $\mathrm{S}$ & $\mathrm{S}$ & $\mathrm{S}$ & $\mathrm{R}$ & $\mathrm{S}$ & $\mathrm{S}$ & $\mathrm{S}$ & $\mathrm{S}$ & $\mathrm{S}$ & 4 \\
\hline $\mathrm{S}$ & $\mathrm{S}$ & $\mathrm{S}$ & $\mathrm{S}$ & $\mathrm{I}$ & $\mathrm{R}$ & $\mathrm{S}$ & $\mathrm{S}$ & $\mathrm{S}$ & $\mathrm{S}$ & $\mathrm{S}$ & $\mathrm{S}$ & $\mathrm{S}$ & $\mathrm{S}$ & $\mathrm{S}$ & 4 \\
\hline
\end{tabular}

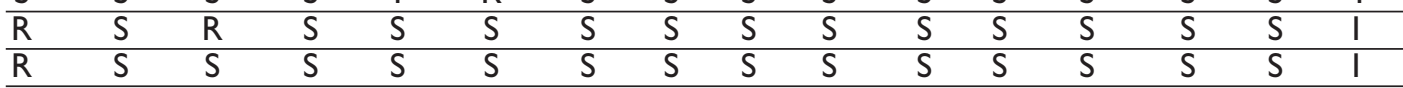

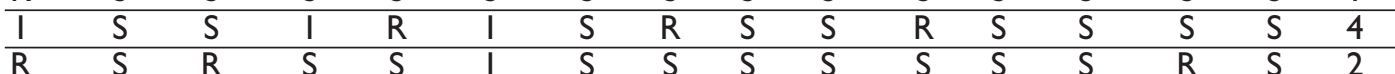

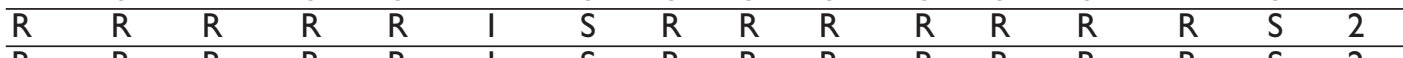

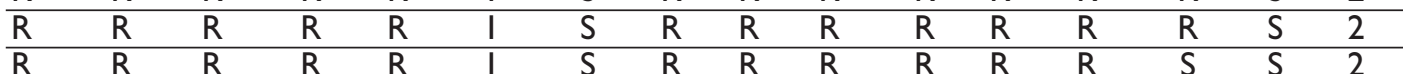

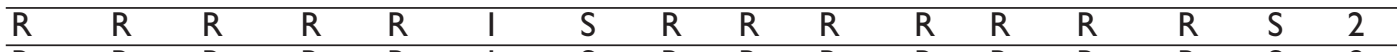

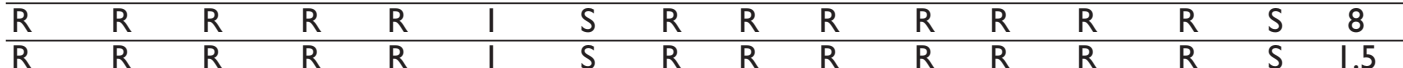

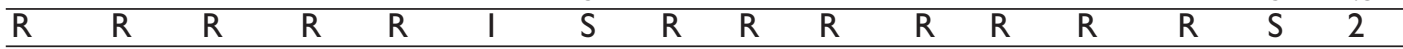

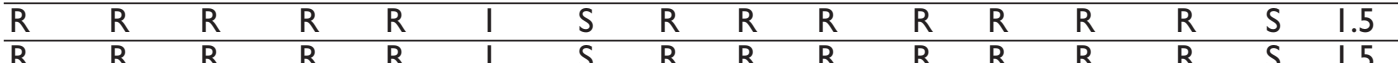

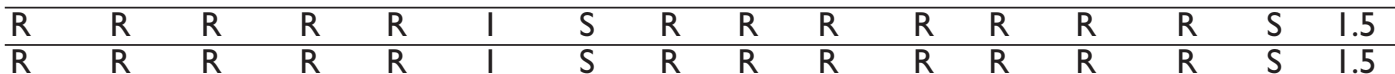

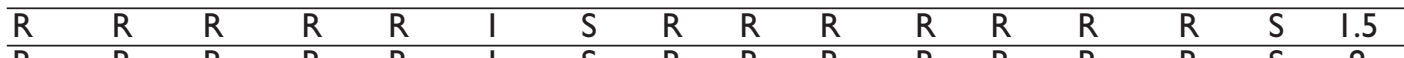

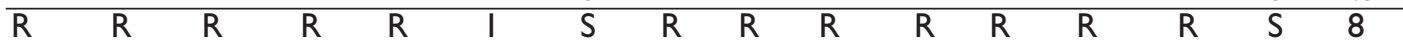

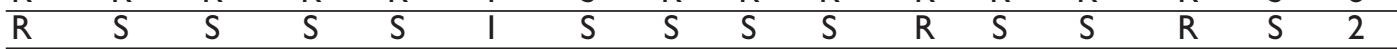
C3

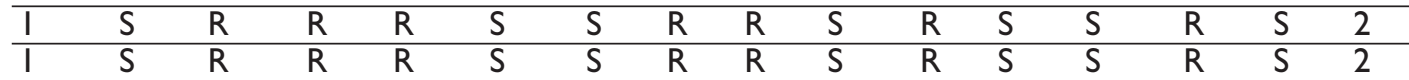

\begin{tabular}{|c|c|c|c|c|c|c|c|c|c|c|c|c|c|c|c|}
\hline $\mathrm{R}$ & $\mathrm{R}$ & $\mathrm{R}$ & $\mathrm{R}$ & $\mathrm{R}$ & $\mathrm{R}$ & $S$ & $\mathrm{R}$ & $\mathrm{R}$ & $\mathrm{R}$ & $\mathrm{R}$ & $\mathrm{R}$ & $\mathrm{R}$ & $\mathrm{R}$ & $\mathrm{S}$ & 2 \\
\hline$\overline{\mathrm{R}}$ & $\mathrm{R}$ & $\mathrm{R}$ & $\mathrm{R}$ & $\mathrm{R}$ & I & S & $\mathrm{R}$ & $\mathrm{R}$ & $\mathrm{R}$ & $\mathrm{R}$ & $\mathrm{R}$ & $\mathrm{R}$ & $\mathrm{R}$ & S & 16 \\
\hline$\overline{\mathrm{R}}$ & $\mathrm{R}$ & $\mathrm{R}$ & $\mathrm{R}$ & $\bar{R}$ & $\mathrm{R}$ & $\mathrm{S}$ & $\mathrm{R}$ & $\mathrm{R}$ & $\mathrm{R}$ & $\mathrm{R}$ & $\mathrm{R}$ & $\mathrm{R}$ & $\mathrm{R}$ & $\mathrm{S}$ & 2 \\
\hline $\mathrm{R}$ & $\mathrm{R}$ & $\mathrm{R}$ & $\mathrm{R}$ & $\mathrm{R}$ & $\mathrm{R}$ & S & $\mathrm{R}$ & $\mathrm{R}$ & $\mathrm{R}$ & $\mathrm{R}$ & $\mathrm{R}$ & $\mathrm{R}$ & $\mathrm{R}$ & $S$ & 1.5 \\
\hline $\mathrm{R}$ & $\mathrm{R}$ & $\mathrm{R}$ & $\mathrm{R}$ & $R$ & 1 & $S$ & $\mathrm{R}$ & $\mathrm{R}$ & $\mathrm{R}$ & $\mathrm{R}$ & $\mathrm{R}$ & $\mathrm{R}$ & $\mathrm{R}$ & $\mathrm{S}$ & 1.5 \\
\hline $\bar{R}$ & $\mathrm{R}$ & $\mathrm{R}$ & $\mathrm{R}$ & $\mathrm{R}$ & $\mathrm{R}$ & $\mathrm{S}$ & $\mathrm{R}$ & $\mathrm{R}$ & $\mathrm{R}$ & $\mathrm{R}$ & $\mathrm{R}$ & $\mathrm{R}$ & $\mathrm{R}$ & S & 1.5 \\
\hline $\mathrm{R}$ & $\mathrm{R}$ & $\mathrm{R}$ & $\mathrm{R}$ & $\mathrm{R}$ & I & S & $\mathrm{R}$ & $\mathrm{R}$ & $\mathrm{R}$ & $\mathrm{R}$ & $\mathrm{R}$ & $\mathrm{R}$ & $\mathrm{R}$ & S & I \\
\hline$\overline{\mathrm{R}}$ & $\mathrm{R}$ & $\mathrm{R}$ & $\mathrm{R}$ & $\mathrm{R}$ & I & $\mathrm{S}$ & $\mathrm{R}$ & $\mathrm{R}$ & $\mathrm{R}$ & $\mathrm{R}$ & $\mathrm{R}$ & $\mathrm{R}$ & $\mathrm{R}$ & $\mathrm{S}$ & 2 \\
\hline $\bar{R}$ & $\mathrm{R}$ & $\mathrm{R}$ & $\mathrm{R}$ & $R$ & 1 & $\mathrm{~S}$ & $\mathrm{R}$ & $\mathrm{R}$ & $R$ & $\mathrm{R}$ & $\mathrm{R}$ & $\mathrm{R}$ & $\mathrm{R}$ & $\mathrm{S}$ & 1.5 \\
\hline $\mathrm{R}$ & $\mathrm{R}$ & $\mathrm{R}$ & $\mathrm{R}$ & $\mathrm{R}$ & $\mathrm{R}$ & S & $\mathrm{R}$ & $\mathrm{R}$ & $\mathrm{R}$ & $\mathrm{R}$ & $\mathrm{R}$ & $\mathrm{R}$ & $\mathrm{R}$ & $S$ & 1.5 \\
\hline$\overline{\mathrm{R}}$ & $\mathrm{R}$ & $\mathrm{R}$ & $\mathrm{R}$ & $\mathrm{R}$ & I & $\mathrm{S}$ & $S$ & $S$ & $\mathrm{R}$ & $\mathrm{R}$ & $\mathrm{R}$ & $\mathrm{R}$ & $\mathrm{S}$ & $\mathrm{S}$ & 2 \\
\hline$\overline{\mathrm{R}}$ & $\mathrm{R}$ & $\mathrm{R}$ & $\mathrm{R}$ & $\bar{R}$ & $\mathrm{R}$ & $\mathrm{S}$ & $\mathrm{R}$ & $\mathrm{R}$ & $\mathrm{R}$ & $\mathrm{R}$ & $\mathrm{R}$ & $\mathrm{R}$ & $\mathrm{R}$ & $\mathrm{S}$ & 1.5 \\
\hline $\mathrm{R}$ & $\mathrm{R}$ & $\mathrm{R}$ & $\mathrm{R}$ & $\mathrm{R}$ & $\mathrm{R}$ & $S$ & $\mathrm{R}$ & $\mathrm{R}$ & $\mathrm{R}$ & $R$ & $\mathrm{R}$ & $\mathrm{R}$ & $\mathrm{R}$ & $S$ & 1.5 \\
\hline$\overline{\mathrm{R}}$ & $\mathrm{R}$ & $\mathrm{R}$ & $\mathrm{R}$ & $\mathrm{R}$ & I & $S$ & $\mathrm{R}$ & $\mathrm{R}$ & $\mathrm{R}$ & $\mathrm{R}$ & $\mathrm{R}$ & $\mathrm{R}$ & $\mathrm{R}$ & $\mathrm{S}$ & 1.5 \\
\hline$\overline{\mathrm{R}}$ & $\mathrm{R}$ & $\mathrm{R}$ & $\mathrm{R}$ & $\bar{R}$ & I & $S$ & $\mathrm{R}$ & $\mathrm{R}$ & $\mathrm{R}$ & $\mathrm{R}$ & $\mathrm{R}$ & $\mathrm{R}$ & $\mathrm{R}$ & $\mathrm{S}$ & I \\
\hline $\mathrm{I}$ & $S$ & $S$ & $\mathrm{R}$ & $\mathrm{R}$ & $\mathrm{R}$ & $S$ & $\mathrm{R}$ & $S$ & $S$ & $\mathrm{R}$ & $\mathrm{S}$ & $\mathrm{S}$ & $\mathrm{R}$ & $S$ & 4 \\
\hline$\overline{\mathrm{S}}$ & $\mathrm{S}$ & $\mathrm{S}$ & $\mathrm{S}$ & $S$ & $\mathrm{~S}$ & $\mathrm{~S}$ & $S$ & $S$ & $\mathrm{~S}$ & $\mathrm{~S}$ & $\mathrm{~S}$ & $\mathrm{~S}$ & $\mathrm{~S}$ & $\mathrm{~S}$ & 0.75 \\
\hline $\mathrm{S}$ & $\mathrm{S}$ & $\mathrm{S}$ & $\mathrm{S}$ & $S$ & $\mathrm{~S}$ & $\mathrm{~S}$ & $S$ & $\mathrm{~S}$ & $\mathrm{~S}$ & $\mathrm{~S}$ & $\mathrm{~S}$ & $\mathrm{~S}$ & $\mathrm{~S}$ & $\mathrm{~S}$ & 0.5 \\
\hline $\mathrm{R}$ & $\mathrm{R}$ & $\mathrm{R}$ & $\mathrm{R}$ & $\mathrm{R}$ & $\mathrm{R}$ & $\mathrm{S}$ & $S$ & $\mathrm{R}$ & $\mathrm{R}$ & $\mathrm{R}$ & $S$ & I & $\mathrm{R}$ & $S$ & 0.5 \\
\hline$\overline{\mathrm{S}}$ & $\mathrm{S}$ & $\mathrm{I}$ & $\mathrm{S}$ & $S$ & $\mathrm{~S}$ & $S$ & $S$ & $\mathrm{~S}$ & $\mathrm{~S}$ & $\mathrm{~S}$ & $\mathrm{~S}$ & $\mathrm{~S}$ & $\mathrm{~S}$ & $\mathrm{~S}$ & 0.5 \\
\hline$\overline{\mathrm{R}}$ & $\mathrm{S}$ & $\mathrm{R}$ & $\mathrm{S}$ & $S$ & $\mathrm{~S}$ & $S$ & $S$ & $\mathrm{~S}$ & $\mathrm{~S}$ & $\mathrm{~S}$ & $\mathrm{~S}$ & $\mathrm{~S}$ & $\mathrm{~S}$ & $\mathrm{~S}$ & 1.5 \\
\hline$\overline{\mathrm{R}}$ & $\mathrm{S}$ & $\mathrm{S}$ & $\mathrm{R}$ & $S$ & $\mathrm{~S}$ & $\mathrm{~S}$ & $S$ & $\mathrm{~S}$ & $\mathrm{~S}$ & $\mathrm{~S}$ & $\mathrm{~S}$ & $\mathrm{~S}$ & $\mathrm{~S}$ & $\mathrm{~S}$ & 1.5 \\
\hline$\overline{\mathrm{R}}$ & $\mathrm{S}$ & $\mathrm{R}$ & $\mathrm{S}$ & $S$ & $\mathrm{~S}$ & $\mathrm{~S}$ & $S$ & $\mathrm{~S}$ & $\mathrm{~S}$ & $\mathrm{~S}$ & $\mathrm{~S}$ & $\mathrm{~S}$ & $\mathrm{~S}$ & $\mathrm{~S}$ & I \\
\hline $\mathrm{R}$ & $\mathrm{R}$ & $\mathrm{R}$ & $\mathrm{R}$ & $\mathrm{R}$ & I & $\mathrm{S}$ & $\mathrm{R}$ & $\mathrm{R}$ & $\mathrm{R}$ & $\mathrm{R}$ & $\mathrm{R}$ & $\mathrm{R}$ & $\mathrm{R}$ & S & I \\
\hline
\end{tabular}
Legenda: AMC, amoxicillina-aido clavulanico;TZP, piperacillina-tazobactam; CRO, ceftriaxone; FEP, cefepime; CAZ, ceftazidime;AZT, aztreonam, SAM, ampicillina-sulbactam; G, gentamicina; AK, amikacina; CIP, ciprofloxacina; SXT, cotrimossazolo; IP, imipenem; MEM, meropenem; TOB, tobramicina; CS, colistina; tgc, tigeciclina. 
isolati da cinque soggetti ricoverati presso le Unità di Rianimazione $(\mathrm{n}=2)$, Chirurgia, Medicina e Pneumologia. C2 include cinque ceppi indistinguibili con un'average similarity ratio di $97.1 \%$, senza differenze nelle bande isolati da cinque pazienti degenti presso le Unità di Rianimazione $(\mathrm{n}=3)$, Stroke Unit, Riabilitazione e Neurologia. Infine, $\mathrm{C} 3$ include trenta stipiti microbici indistinguibili (average similarity ratio di 95\%): il 67\% di questi ceppi era isolato da pazienti ammessi in Rianimazione.

Tutti i ceppi inclusi nel cluster $\mathrm{C} 3$ sono risultati indistinguibili dall'European clone II.

In tutti i tre cluster il caso "sorgente" è stato isolato da pazienti degenti in Rianamazione. Il ceppo costituente il cluster $\mathrm{A}_{1}$ apparteneva all'European clone I, mentre nessuno dei ceppi era riconducibile all'European clone III.

Gli isolati erano considerati colonizzanti salvo che in due casi in cui si sono resi responsabili di batteriemia.

I risultati dei test di antibioticosensibilità sono compendiati nella Tabella 1, da cui è possibile riconoscere quattro differenti profili: il primo raggruppa ceppi sensibili alla più parte degli antimicrobici testati; il secondo comprende stipiti sensibili ai carbapenemi, a colistina e ad ampicillinasulbactam; il terzo è costituito da ceppi sensibili agli aminoglucosidi, a colistina e ad ampicillinasulbactam, ed infine il quarto profilo si caratterizza per la sensibilità esclusivamente a colistina e ad ampicillina-sulbactam.

Le MIC per tigeciclina erano comprese tra 0.5 $\mathrm{mg} / \mathrm{L}$ e $16 \mathrm{mg} / \mathrm{L}$ con $\mathrm{MIC}_{50}$ e $\mathrm{MIC} \mathrm{C}_{90}$ di $1.5 \mathrm{mg} / \mathrm{L} \mathrm{e}$ $4 \mathrm{mg} / \mathrm{L}$ rispettivamente.

\section{DISCUSSIONE}

Acinetobacter spp. è un coccobacillo Gram negativo che in anni recenti è stato sempre più frequentemente identificato quale causa di infezioni associate alle organizzazioni sanitarie $(5,11)$.

Considerato commensale con un basso livello di patogenicità, mostra tuttavia un'eccellente capacità di acquisire resistenze gli antibiotici ed a permanere nell'ambiente $(11-12,16)$. Acinetobacter spp. è responsabile di più del $10 \%$ di tutte le infezioni da batteri Gram negativi nelle Unità di Terapia Intensiva in Europa e negli Stati Uniti di America $(6,11,14,16)$.

I sistemi di sorveglianza delle infezioni ospedaliere devono essere efficienti nell'identificazione di tali eventi (8-10). In questa prospettiva la tecnica di rep-PCR appare essere altamente efficiente nel definire precocemente la circolazione clonale di microrganismi epidemici, confermando od escludendo correlazioni genetiche tra i ceppi isolati (4). In questo studio il sistema di rep-PCR Diversilab ${ }^{\mathrm{TM}}$ ha identificato diciannove diversi pattern di Acinetobacter spp. ed ha confermato l'esistenza di tre focolai epidemici, tutti a partenza dall'Unità di Rianimazione e poi traslocati in altri reparti di diagnosi e cura.

Il cluster principale era costituito da batteri appartenenti all'European clone II (49.2\% dei ceppi) e solo in un caso il ceppo era del clone Europa I.

Non è stata rilevata alcuna correlazione tra fingerprints e tipologia di campione biologico di origine, né tra fingerprints e quadro di gravità clinica.

L'emergenza dei ceppi di Acinetobacter multiresistenti è un fenomeno sempre più attuale che si caratterizza per la difficoltà nell'impostazione di un'efficace opzione terapeutica soprattutto nel caso di ceppi resistenti ai carbapenemi la cui conferma deve essere effettuata mediante la determinazione della Minima Concentrazione Inibente mediante metodo di diluizione in brodo, diluizione in agar oppure E-test.

Alcuni inibitori delle beta-lattamasi, come Tazobactam e Acido clavulanico, sono stati utilizzati con successo in combinazione con un betalattamico nel trattamento delle infezioni da Acinetobacter spp.

Tra queste molecole, Sulbactam presenta una duplice azione agendo come inibitore delle betalattamasi e possedendo un'attività antimicrobica intrinseca nei confronti di Acinetobacter spp.

Numerosi studi in vitro suggeriscono inoltre che l'associazione Ampicillina-Sulbactam risulta costantemente più efficace nei confronti di Acinetobacter spp. rispetto alla maggior parte delle cefalosporine, Ciprofloxacina, Gentamicina e delle altre combinazioni beta-lattamico-inibitore delle beta-lattamasi.

Questa maggiore efficacia sembra essere dovuta al solo Sulbactam, come confermano alcuni studi su modello animale in cui Sulbactam in monoterapia ha presentato maggiore efficacia rispetto ad altre betalattamine su Acinetobacter spp.

Inoltre, il $66.2 \%$ dei ceppi isolati risultava resistente ai carbapenemi. Tuttavia, la Colistina e l'Ampicillina-Sulbactam mostravano la migliore attività in vitro confermandosi la migliore opzione terapeutica.

Il metodo di rep-PCR è risultato altamente efficace e specifico ed ha permesso di individuare un focolaio epidemico all'interno del reparto di Rianimazione. Il sistema ha permesso inoltre una suddivisione specifica degli isolati analizzati e di individuare cluster di strains geneticamente più simili tra loro.

Tale metodo è risultato inoltre utile per una rapida classificazione dei ceppi quando comparati con $\mathrm{i}$ ceppi di riferimento. 


\section{BIBLIOGRAFIA}

1. Bergogne-Berezin E, Joly-Guillou ML. An underestimated nosocomial pathogen, Acinetobacter calcoaceticus. J Antimicrob Chemother 1985; 16: 535-8.

2. Bergogne-Berezin E, Joly-Guillou ML, Vieu JF. Epidemiology of nosocomial infections due to Acinetobacter calcoaceticus. J Hosp Infect 1987; 10: 105-13.

3. Brown S, Amyes S. OXA (beta)-lactamases in Acinetobacter: the story so far. $J$ Antimicrob Chemother 2006; 57: 1-3.

4. Carretto E, Barbarini D, Farina C, Grosini A, Nicoletti $\mathrm{P}$, Manso E. Use of the DiversiLab ${ }^{\mathbb{R}}$ semiautomated repetitive-sequence-based polymerase chain reaction for epidemiologic analysis on Acinetobacter baumannii isolates in different Italian hospitals. Diagn Microbiol Infect Dis 2008; 60: 1-7.

5. Dijkshoorn L, Nemec A, Seifert H. An increasing threat in hospitals: multidrugresistant Acinetobacter baumannii. Nat Rev Microbiol 2007; 5: 939-51.

6. Hanberger H, Garcia-Rodriguez JA, Gobernado M, Goossens H, Nilsson LE, Struelens MJ. Antibiotic susceptibility among aerobic Gram negative bacilli in intensive care units in 5 European countries. French and Portuguese ICU Study Groups. JAMA 1999; 281: 67-71.

7. Kramer A, Schwebke I, Kampf G. How long do nosocomial pathogens persist on inanimate surfaces? A systematic review. BMC Infect Dis 2006; 6:130.

8. Kristóf K, Szabó D, Marsh JW, et al. Extended-spec- trum beta-lactamase-producing Klebsiella spp. in a neonatal intensive care unit: risk factors for the infection and the dynamics of the molecular epidemiology. Eur J Clin Microbiol Infect Dis 2007; 26: 563-70.

9. Lipworth AD, Hyle EP, Fishman NO, et al. Limiting the emergence of extended-spectrum beta-lactamaseproducing Enterobacteriaceae: influence of patient population characteristics on the response to antimicrobial formulary interventions. Infect Control Hosp Epidemiol 2006; 27: 279-86.

10. Paterson DL. Resistance in Gram negative bacteria: Enterobacteriaceae. Am J Med 2006; 119: S20-S28 discussion S62-70.

11. Peleg AY, Seifert H, Paterson DL. Acinetobacter baumannii: emergence of a successful pathogen. Clin Microbiol Rev 2008; 21: 538-82.

12. Perez F, Hujer AM, Hujer KM, Decker BK, Rather $\mathrm{PN}$, Bonomo RA. Global challenge of multidrugresistant Acinetobacter baumannii. Antimicrob Agents Chemother 2007; 51: 3471-84.

13. Retailliau HF, Hightower AW, Dixon RE, Allen JR. Acinetobacter calcoaceticus: a nosocomial pathogen with an unusual seasonal pattern. J Infect Dis 1979; 139: 371-5.

14. Richet H, Fournier PE. Nosocomial infections caused by Acinetobacter baumannii: a major threat worldwide. Infect Control Hosp Epidemiol 2006; 27: 645-6.

15. Van Looveren M, Goossens. HARPAC Steering Group. Antimicrobial resistance of Acinetobacter spp. in Europe. Clin Microbiol Infect 2004; 10: 684-704. 